\title{
Biodentine Induce Proteínas Apoptóticas Caspasa 3 y PARP-1 en Células de la Pulpa Dental Humana
}

\author{
Biodentine Induces Apoptotic Proteins Caspase 3 \\ and PARP -1 on Human Dental Pulp Cells
}

\author{
Pablo. F. Romero ${ }^{1,2,4}$; Mario E. Flores²; Pamela Ehrenfeld ${ }^{3,4}$; Tania Köning ${ }^{3}$ \& Francisca Pavicic 3,4
}

ROMERO, P. F.; FLORES, M. E.; EHRENFELD, P.; KÖNING, T. \& PAVICIC, F. Biodentine induce proteínas apoptóticas caspasa 3 y parp-1 en células de la pulpa dental humana. Int. J. Odontostomat., 13(4):411-417, 2019.

RESUMEN: Las patologías pulpares han sido un verdadero reto para la odontología principalmente por su tratamiento. Actualmente, existen numerosos biomateriales en el mercado que reportan tener propiedades inherentes en los tejidos dentarios. Sin embargo, diferentes estudios sobre múltiples líneas celulares expuestas a estos biomateriales demuestran resultados controversiales como biocompatiblidad y citotoxicidad celular. Biodentine, es un cemento endodóntico en base a silicatos cálcico de múltiples aplicaciones, que prestaría propiedades de biocompatibilidad como bioactividad celular, características que le permitirían incluso ser utilizado en contacto directo con la pulpa dental. El objetivo de este estudio es la evaluación in-vitro de Biodentine, sobre cultivos de células de la pulpa dental humana (CCPDH). Se prepararon discos de cemento de Biodentine ${ }^{\mathrm{TM}}$ de $2 \times 6 \mathrm{~mm}$, los que se expusieron a cultivos de células aisladas de la pulpa dental humana. Luego de 24, 48 y 72 horas de exposición, se realizaron ensayos de viabilidad celular utilizando el método colorimétrico MTT. También se realizaron ensayos de expresión proteica de dos proteínas involucradas en la vía de señalización de la apoptosis celular: Caspasa - 3 clivada y Poli(ADP-Ribosa) Polimerasa, PARP - 1. Existen diferencias estadísticamente significativas $(p<0,05)$ en los ensayos de viabilidad celular entre las células expuestas a Biodentine y el grupo control, como también a medida que aumenta el tiempo de exposición $(p<0,05)$. Por otra parte, también existen diferencias significativas $(p<0,05)$ en la expresión de PARP- 1 en los grupos sometidos a Biodentine. Los resultados obtenidos en este estudio demuestran que Biodentine genera citotoxicidad celular en cultivos celulares de pulpa dental humana, por disminución de la viabilidad celular como por la expresión de proteínas apoptóticas. Es por esto que la utilización de este biomaterial debería ser estudiado y considerarse en cada caso clínico, especialmente como recubridor pulpar directo.

PALABRAS CLAVE: citotoxicidad, biodentine, caspasa 3, poli(adp-ribosa) polimerasa, células de la pulpa dental humana.

\section{INTRODUCCIÓN}

El complejo pulpo dentinario es afectado por un sinnúmero de estímulos nocivos que provocan inflamación, muerte o distrofia pulpar. Estos pueden ser de origen bacteriano, traumáticos, iatrogénicos, químicos e idiopáticos. Los más comunes son procesos infecciosos, siendo el más prevalente la enfermedad de caries, donde la inflamación pulpar es generada por la invasión bacteriana, la liberación de proteasas y metabolitos (Cohen et al., 2011).

Actualmente, para el tratamiento de estas patologías, existen diferentes biomateriales que presentan pro- piedades mecánicas similares a los tejidos dentarios, biocompatibilidad, bioactividad celular, entre otros (Jung et al., 2015; Chang et al., 2015). Recientemente, un nuevo cemento de relleno pulpar en base a silicatos de calcio, (CSC) es comercializado bajo la marca Biodentine (Septodont. Saint-Maur-des-Fossés, France) (Chang et al., 2014) el cual no presentaría los inconvenientes que exhiben otros CSC, tales como propiedades mecánicas poco similares a la dentina, tiempo de fraguado inicial tardío y citotoxicidad. De acuerdo al fabricante, el cemento Biodentine se prepara a partir de un polvo y un líquido, previamente dosificados por el fabricante. La

\footnotetext{
${ }^{1}$ Escuela de Odontología, Facultad de Medicina, Universidad Austral de Chile, Valdivia, Chile.

2 Instituto de Ciencias Químicas, Facultad de Ciencias, Universidad Austral de Chile, Valdivia, Chile.

${ }^{3}$ Instituto de Anatomía, Histología y Patología, Facultad de Medicina, Universidad Austral de Chile, Valdivia, Chile.

${ }^{4}$ Programa Honor de Investigación de la Facultad de Medicina, PHIM, Universidad Austral de Chile, Valdivia, Chile.
} 
porción sólida contiene silicato tricálcico como agente potencialmente bioactivo (Camilleri et al., 2013), carbonato de calcio y oxido de zirconio como radiopacificador; mientas que el líquido contiene una solución de clorhidrato de calcio, aceleradores y agentes reductores. Este CSC es recomendado como sustitutos dentinarios, en restauraciones coronales, reparación de perforaciones y además como recubridor pulpar, el que luego de aplicado podría estar en contacto directo con la pulpa vital (Rathinam et al., 2016; Emara et al., 2018).

Hay estudios que muestran las propiedades fisicoquímicas y biológicas de Biodentine, específicamente la biocompatibilidad sobre diferentes líneas celulares como en cultivos celulares de la pulpa dental humana (CCPDH). Estos estudios muestran que Biodentine induce la mineralización temprana de la pulpa dental (Jung et al.) al igual que el aumento de la viabilidad celular comparada con otros materiales en base a silicatos. Por el contrario, existe evidencia de que este CSC es citotóxico a nivel celular, ya que se ha visto la expresión de citoquinas pro inflamatorias y factor de necrosis tumoral alfa (TNF- $\alpha$ ), las cuales están involucradas en la respuesta inflamatoria inicial, en la producción de especies reactivas de oxígeno (EROs), como también en actividad necrótica y apoptótica (Chang et al., 2014).

Algunos componentes citotóxicos de los CSC podrían afectar la viabilidad de las células de la pulpa dental y causar muerte celular por necrosis o apoptosis (Akbulut et al., 2018). La apoptosis puede desencadenarse en primera instancia por un daño irreparable del ADN, aberraciones en el metabolismo celular, glucocorticoides y EROs (Nikitakis et al., 2004).

La vía de señalización de la muerte celular programada es llevada a cabo por múltiples uniones receptor-ligando y por proteínas específicas que actúan en cadena. Una de ellas es la caspasa-3, la cual es un punto común entre la vía extrínseca e intrínseca de la señalización apoptótica. Esta última está mediada por los receptores TNRF1 y activado por TNF - $\alpha$ (Sosna et al., 2014). Otras proteínas son las Poli - ADP - ribosa Polimerasa (PARP), entre las que se encuentra PARP-1 encargada de unirse al ADN y detectar daños para desencadenar diferentes procesos metabólicos como el de muerte celular (Boulares et al., 1999; Wang et al., 2015). Se ha visto, en cultivos celulares en presencia de Biodentine, la presencia de EROs, citoquinas pro- inflamatorias como también la presencia de células apoptóticas (Chang et al., 2014). A la fecha no se ha encontrado evidencia que demuestre la expresión de proteínas apoptóticas específicas, por lo tanto es importante examinar el mecanismo de muerte celular que los CSC podrían inducir.

El propósito del presente estudio es evaluar el efecto citotóxico del cemento Biodentine, y la capacidad de inducir apoptosis sobre cultivos celulares de células de la pulpa dental humana.

\section{MATERIAL Y MÉTODO}

Cultivo celular. Los cultivos celulares de la pulpa dental humana fueron obtenidos a través de cultivos primarios de pulpa dental. Estas fueron crecidas en medio Dulbecco's Modified Eagle Medium (DMEM, 1.8 $\mathrm{mM} \mathrm{Ca}^{+2}$ DMEM, Hyclone), suplementado con suero bobino fetal al $10 \%$ (SBF, Gibco), penicilinaestreptomicina al $1 \%(10000 \mathrm{Ul} / \mathrm{ml}$ de penicilina G sódica y $10000 \mu \mathrm{g} / \mathrm{ml}$ de sulfato de estreptomicina; Gibco) y Fungizone $\AA$ al $1 \%(250 \mu \mathrm{g} / \mathrm{ml}$ anfotericina B; Gibco). El cultivo se llevó a cabo a $37^{\circ} \mathrm{C}$ con un 5 $\%$ de $\mathrm{CO}_{2}$, hasta subconfluencia.

Preparación de discos de cemento. Se preparó los discos de Biodentine bajo condiciones asépticas y en cámara de flujo laminar, acorde a las instrucciones entregadas por el fabricante. La mezcla obtenida se depositó en moldes de $6 \mathrm{~mm}$ de diámetro por $2 \mathrm{~mm}$ de altura (Chang et al., 2015). Se dejó la mezcla en los moldes en cámara de flujo laminar por 24 horas. Previo al cultivo con células, se esterilizó los discos por radiación ultravioleta.

Ensayo de Viabilidad celular por MTT. Este ensayo se basa en la reducción metabólica del Bromuro de 3-(4,5-dimetiltiazol-2-ilo)-2,5-difeniltetrazol (MTT), realizada por la enzima mitocondrial succinatodeshidrogenasa, en un compuesto coloreado de color azul (Formazano). Para llevar a cabo este ensayo, se sembró $3 \times 10^{5}$ células de pulpa dental humana por pocillo, las que fueron mantenidas en DMEM al $10 \%$ suplementado con SBF. A continuación, se introdujo los discos estériles de Biodentine. Transcurrido un tiempo determinado de exposición al CSC (24, 48 y 72 horas), se procedió a retirar tanto el medio de cultivo como el disco de cemento. Se adicionó a la placa de cultivo $100 \mu \mathrm{l}$ de una solución de MTT de concentración 0,55 mg/mL (Sigma Aldrich, 
St. Louis, MO,USA), dejándose en contacto con las células durante 4 horas. Luego se procedió a solubilizar el formazano en $1 \mathrm{~mL}$ de una mezcla de isopropanol y ácido clorhídrico en proporción 2:10 v/v (Merck KGaA, Darmstadt, Alemania) para luego medir la absorbancia a $570 \mathrm{~nm}$, utilizando un espectrofotómetro (Bio-MateTM 3S Waltham, MA USA, Thermo Fisher Scientific Inc.). El porcentaje de viabilidad celular se obtuvo siguiendo las normas para la evaluación biológica de productos médicos, mediante ensayos de citotoxicidad por MTT (Li et al., 2015) (ISO 10993-5). Todos los ensayos fueron realizados por triplicado.

Western Blot. Transcurrido un tiempo de exposición al cemento (24, 48 y 72 horas), se procedió a obtener extractos proteicos totales. Los discos de Biodentine fueron removidos del medio de cultivo, para luego agregar $100 \mu \mathrm{L}$ de tampón RIPA (50 mM Tris- $\mathrm{HCl}$ pH 7,5, $150 \mathrm{mM} \mathrm{NaCl}, 1 \%$ Nonidet P-40, 1 mM EDTA, $1 \mathrm{mM}$ EGTA) conteniendo una mezcla de inhibidores $(2,5$ $\mathrm{mM} / \mathrm{ml}$ de fosfato de nitrofenilo, $1 \mathrm{mM}$ de fluoruro de fenilmetano-sulfonilo, $1 \mathrm{mM} / \mathrm{ml}$ de aprotinina, leupeptina y pepstatina, $1 \mathrm{mM} \mathrm{NaF}$ y $1 \mathrm{mM} \mathrm{Na}_{3} \mathrm{VO}_{4}$ ), de tal forma de detener la reacción. Las células fueron raspadas y sonicadas de las placas de cultivo, para luego determinar la cantidad de proteínas mediante el método de Bradford. Las muestras fueron sometidas a geles de electroforesis SDS-PAGE y electrotransferidas a una membrana de Inmobilon $P \circledR$ (Sigma Aldrich, Saint Louis, Missouri, USA), las que fueron incubadas con anticuerpo primario para caspasa-3, caspasa-3 clivada y PARP-1 (Cell Signaling Technology, Danvers, MA, USA), y anticuerpo secundario de ratón y conejo conjugado a HRP (Cell Signaling Technology, Danvers, MA, USA) según la procedencia del primer anticuerpo. Las bandas inmunorreactivas se visualizaron mediante un kit de quimioluminiscencia (Thermo Fisher Scientific Inc.MA USA). Para la visualización densitométrica de las bandas inmunorreactivas, se utilizó una medidor de alto rendimiento para quimioluminiscencia (G: Box Chemi XX8, Syngene, Synoptic Ltd. Cambridge, UK). El posterior análisis de las bandas se realizó a través del software Image J (versión 1.50i). Los datos fueron graficados en el programa Statgraphics Centurion XV.II (versión 15.02.0006).

Análisis Estadístico. Se aplicó el test estadístico TStudent en el análisis de viabilidad celular y expresión de proteínas, de tal forma de establecer diferencias entre los mismos tiempos de exposición entre los cultivos celulares que fueron sometidos al tratamiento $y$ los que no. Para establecer diferencias entre los grupos y a diferentes tiempos de exposición, se aplicó el test estadístico ANOVA simple y posterior aplicación de test Tukey. El nivel de significancia estadística se determinó al $5 \%$.

\section{RESULTADOS}

Citotoxicidad de Biodentine. Biodentine muestra citotoxicidad celular a medida que aumenta el tiempo de exposición en los cultivos celulares (Fig. 1). Cada uno de los grupos presenta diferencias estadísticamente significativas con su grupo control respectivo $(p<0,05)$. El grupo de las 24 con el de 72 horas presentan diferencias estadísticamente significativas $(p<0,05)$. No existen diferencias entre el grupo de 24 con el de 48 horas, al igual que entre el grupo de 72 con el de 48 horas.

Efecto de Biodentine en la vía de señalización apoptótica. El tratamiento de los cultivos celulares en presencia del cemento Biodentine induce la expresión de las proteínas apoptóticas a medida que aumenta el tiempo de exposición, en comparación con el grupo control, estableciendo diferencias estadísticamente

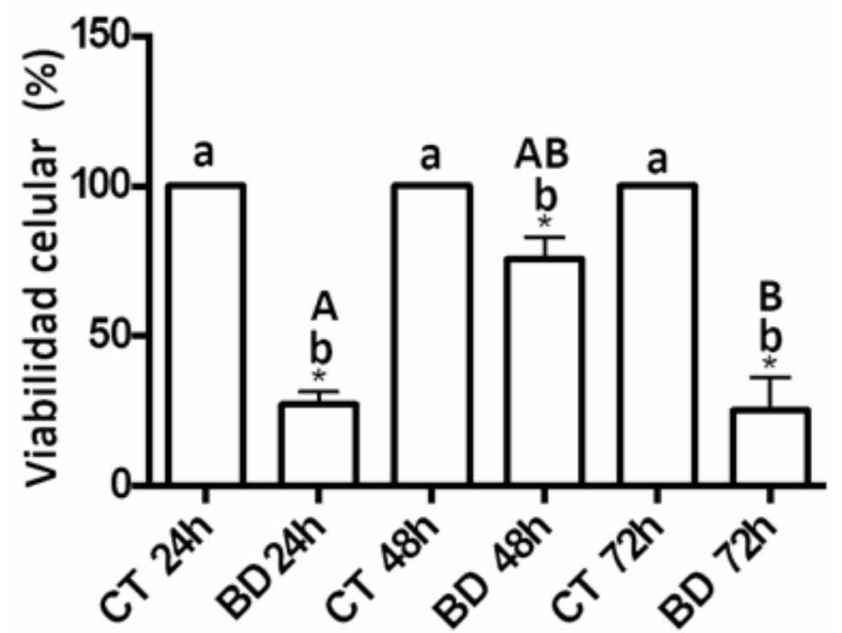

Fig. 1. Ensayo de viabilidad celular por MTT, a diferentes tiempos de exposición, de cultivos expuestos a Biodentine. Todos los controles representan un $100 \%$ de viabilidad ceIular. Letras minúsculas distintas indican diferencias estadísticamente significativas entre tratamientos $(p<0,05)$. Letras mayúsculas distintas indican diferencias estadísticamente significativas entre tiempos de exposición $(p<0,05)$. Barra de error indica desviación estándar. *BD: Biodentine, *CT: control. Eje Y: viabilidad celular porcentual, eje X: tiempo de exposición. Los datos son representativos de 5 experimentos independientes. 


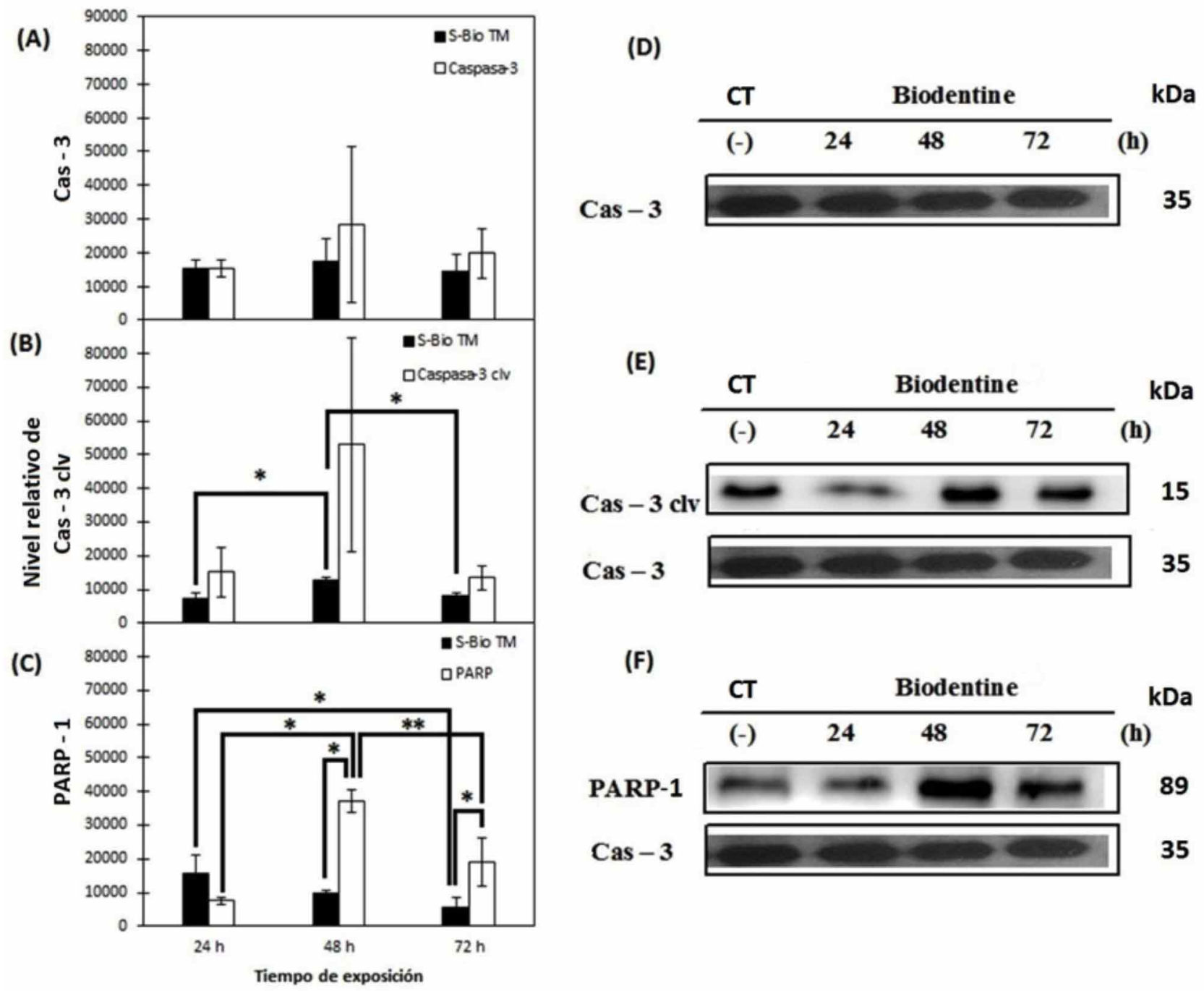

Fig. 2. Efecto de Biodentine en la expresión relativa de (A) caspasa - 3 (Cas - 3) (B) caspasa - 3 clivada (cas - 3 clv) y (C) PARP-1 en células de la pulpa dental humana. Las células fueron cultivadas con Biodentine a 24, 48, y 72 horas (A, B, C, D, E y F). ( $D, E$ y F) representan cinética y nivel relativo de caspasa - 3, caspasa - 3 clivada y PARP -1 . Caspasa - 3 (A y D) fue utilizada como control de carga. La expresión proteica fue determinada mediante análisis de Western blot. $\left({ }^{*}\right)$ : Diferencias estadísticamente significativa $(p<0,05) .\left({ }^{* *}\right)$ Diferencia altamente significativa $(p<0,001)$. Barras paralelas al eje $Y$ indican desviación estándar. kDa: kilodalton, CT: control, (h): horas. Los datos son representativos de 5 experimentos independientes.

significativas $(p<0,05)$. La proteína caspasa-3 clivada presenta a las 48 horas la mayor expresión, al igual que PARP-1, en el mismo tiempo. La expresión de la proteína PARP-1 presenta diferencias estadísticamente significativas entre el grupo de 24 y 48 horas $(p<0,05)$, al igual que entre el grupo de 48 y 72 horas $(p<0,05)$. La expresión de la proteína caspasa-3 clivada presenta diferencias estadísticamente significativas entre el grupo de 24 en comparación con el grupo de 48 horas en contacto con Biodentine (Fig. 2). Las imágenes de los Western blots para caspasa-3 y PARP-1 corresponden a cinco experimentos independientes.

\section{DISCUSIÓN}

Biodentine es presentando como un nuevo cemento diseñado para mejorar ciertas falencias que presentaba el cemento MTA, considerado como patrón oro entre los cementos dentales fabricados con silicatos de calcio. La aplicación terapéutica de estos CSC implica una interacción entre los tejidos y el material, el cual estará en contacto directo con la pulpa dental (Emara et al.). Aunque hay reportes que indican que Biodentine es biocompatible y que permite la diferenciación celular (Chang et al., 2015), no queda 
muy clara la participación de estos cementos en la activación de la apoptosis celular, al igual que en la producción de EROs (Jung et al.; Chang et al., 2015). Hasta la actualidad no se ha encontrado literatura que relacione este biomaterial con la expresión de proteínas apoptóticas específicas.

Los resultados de este estudio arrojan una baja viabilidad celular en CCPDH en presencia de Biodentine, en comparación con su grupo control, en los tres tiempos de exposición. Los valores de viabilidad celular entre el grupo control a las 24 horas en comparación con el grupo expuesto al cemento es de un $87,8 \%$, la más alta obtenida en este estudio. La baja viabilidad celular inicial podría deberse al efecto citotóxico del hidróxido de calcio, compuesto liberado de manera abundante e inmediata después de la preparación de Biodentine, que presenta un pico de liberación luego del primer día de preparación (Ozdemir et al., 2008). Sin embargo, la viabilidad celular a las 72 horas de exposición disminuyó considerablemente a $72,8 \%$. Se observó una disminución del $24,4 \%$ en los valores de viabilidad celular en el grupo expuesto 48 horas al CSC, en comparación al grupo control. La viabilidad celular disminuye entre las 48 y 72 horas, en un $24,1 \%$. Estos resultados son consistentes con los obtenidos en otros estudios realizados previamente en CCPDH y fibroblastos gingivales humanos (Chang et al., 2014; A Saberi et al., 2016). Por otro parte, existen estudios realizados sobre CCPDH, donde Biodentine y MTA presentan diferencias significativas en cuanto a citotoxicidad a los 1 y 3 días de cultivo, en comparación al grupo control (Zhou et al., 2013). La citotoxicidad de Biodentine a las 72 horas puede deberse al efecto alcalino de este cemento. De hecho algunos autores demostraron en un análisis a tres días de evaluación, mediante un electrodo selectivo de iones de $\mathrm{Ca}^{+2}$, demostró que Biodentine presenta un pico de alcalinidad $(\mathrm{pH}=9)$, que podría ser un factor que inhiba la activación de enzimas en la membrana citoplasmática de $\mathrm{CCPDH}$, al igual que el hidróxido de calcio (Akbulut et al.). Otros estudios plantean que los ensayos de MTT solo muestran daño celular cuando se presenta un estado apoptótico, es decir, cuando el metabolismo está sustancialmente reducido (Zhou et al.). Sin embargo, el estudio presentado en este trabajo complementa los resultados de MTT con ensayos que permiten determinar la participación de Biodentine en la vía de señalización de la apoptosis.

Existen alternativas para evaluar viabilidad y citoxicidad celular como lo es el análisis de expre- sión proteica y la citometría de flujo, siendo este un método rápido, seguro, de bajo costo y de alta sensibilidad para determinar citotoxicidad (Zhou et al.). En estudios realizados sobre cultivos de fibroblastos gingivales humanos a siete días de exposición a Biodentine, se determinó, a través de citometría de flujo, que la cantidad de células en apoptosis temprana fue de un $10,18 \%$, en comparación con el grupo control que registró sólo un 3,64 \% (Zhou et al.). Se ha observado el mismo patrón en cultivos de células madres de dientes temporales y dientes permanentes (Akbulut et al.). Es por esto que a futuro nuestros resultados pudiesen ser corroborados a través de citometría de flujo, para así determinar el porcentaje de células apoptoticas y la presencia de proteínas especificas involucradas en vía de señalización de la apoptosis.

Biodentine es un biocerámico que, de acuerdo a ciertos estudios, presentaría propiedades ideales como biocompatibilidad y baja citotoxicidad. Hay estudios que muestran la respuesta inflamatoria en $\mathrm{CCPDH}$, donde Biodentine provoca un incremento en los niveles de EROs, cicloxigenasa-2, y prostangladina $\mathrm{E} 2$, al igual que la expresión proteica de TNF- $\alpha$. La expresión de proteínas como la caspasa-3, caspasa-3 clivada y PARP-1, que son moléculas conocidas por estar involucradas en la vía de señalización de la apoptosis, es un indicativo de que Biodentine podría contribuir en procesos patológicos (Nikitakis et al.). La vía extrínseca de la apoptosis está mediada por citoquinas proinflamatorias como la TNF- $\alpha$, la que presenta una relación directa con la activación de las caspasas, específicamente caspasa-8 y caspasa-3 (Fragoso et al., 2014). Por otra parte, se ha reportado una relación entre procesos de señalización apoptóticos, como la necroptopsia, con la expresión de PARP-1 (Sosna et al.). Varios estudios muestran la expresión de citoquinas pro inflamatoria (IL-1 $1 \beta$, IL-6, IL-8, NO, PGE2 y TNF- $\alpha$ ) en cultivos celulares en presencia de Biodentine, donde han reportado niveles similares en la expresión de TNF- $\alpha$ en CCPDH expuestos a Biodentine y otros CSC (Chang et al., 2014, 2015; Jung et al.; Rathinam et al.). Resultados similares se han obtenido utilizando cultivos de osteoblastos y odontoblastos (Bortoluzzi et al., 2015; Chang et al., 2015), los que apoyan los resultados obtenidos en este estudio, donde existe mayor expresión de caspasa-3 clivada y PARP-1 a las 48 y 72 horas de exposición a BD.

Se ha descrito diferentes formas de activación 
de las caspasas, como es el caso del peroxinitrito $\left(\mathrm{NO}_{3}\right)$. $\mathrm{NO}_{3}$ es un radical libre de corta vida media, que está implicado en variados fenómenos biológicos, como transmisión sináptica, citotoxicidad macrofágica y muerte celular (Melino et al., 2000; Simon et al., 2000). Estos procesos pueden ser regulados por la S-nitrosilación de proteínas mediadas por especies de NO, como la señalización proteica de JNK2 y las caspasas 3, 6 y 7 (Sosna et al.). En consecuencia, diferentes estudios han demostrado la producción de $\mathrm{NO}$ en cultivos de CCPDH y odontoblastos expuestos a diferentes CSC, donde la producción de NO a las 72 horas es mayor a la del grupo control (Chang et al., 2014, 2015). Es por esto, al igual que en nuestros resultados, que la expresión de caspasa-3 clivada a las 48 y 72 horas es mayor a las del grupo control, fenómeno que podría estar relacionado con la expresión de EROs.

Dentro de los resultados obtenidos en este estudio se puede dilucidar que caspasa-3 clivada y PARP-1 presentan valores similares de expresión, a las 24, 48 y 72 horas de exposición a Biodentine, con un pico a las 48 horas para luego decaer a las 72 horas. Estos resultados concuerdan con la actividad en cadena de la vía de señalización de la apoptosis, donde caspasa-3 influye directamente en la activación de las PARP (Boulares et al.). Se ha visto esta expresión paralela de ambas proteínas en cáncer colorectal, como en la muerte celular de células hematopoyéticas (Cheng et al., 2012). Por lo tanto, los altos niveles de expresión de caspasa3 clivada y PARP-1 en los grupos tratados con Biodentine, podrían estar asociados a muerte celular. Estos resultados pertenecientes a estudios de citoxicidad celular sugieren que la aplicación de Biodentine sobre tejidos dentales vitales podría generar resultados adversos sobre los tejidos dentales vitales en los que se recomienda su aplicación.

\section{CONCLUSIÓN}

Biodentine exhibe actividad citotóxica como apoptótica sobre cultivos de células de pulpa dental humana. Si bien este estudio demuestra la expresión de proteínas apoptóticas específicas, ensayos previos también han demostrado actividad apoptótica. Por lo tanto, la aplicación clínica debería ser estudiada para cada caso en particular, sobre todo para su aplicación como material de recubrimiento pulpar.

\section{AGRADECIMIENTOS}

Se agradece al Proyecto VIDCA-honor, $\mathrm{N}^{\circ}$ PHIM-2017-06, Apoyos de Dirección de Pregrado, Escuela de Pregrado, Escuela de Odontología, Facultad de Medicina, Universidad Austral de Chile por el financiamiento brindado.

ROMERO, P. F.; FLORES, M. E.; EHRENFELD, P.; KÖNING, T. \& PAVICIC, F. Biodentine induces apoptotic proteins Caspase 3 and PARP -1 on human dental pulp cells. Int. J. Odontostomat., 13(4):411-417, 2019.

ABSTRACT: Oral pathologies have been a real challenge for dentistry, mainly due to its treatment. Currently, there are numerous biomaterials on the market that may present inherent properties in dental tissues. However, studies on multiple cell lines are based on biocompatible results such as biocompatibility and cellular cytotoxicity. Biodentine is endodontic cement based on calcium silicates of multiple applications, which would provide biocompatibility properties as cellular bioactivity, characteristics that will allow it to be used in direct contact with the dental pulp. The objective of this study is the in vitro evaluation of Biodentine, on cultures of cells of the human dental pulp (HDPC). Biodentine cement disks of $2 \times 6 \mathrm{~mm}$ were prepared, and HDPC culture plates were introduced. After 24, 48 and 72 hours of exposure, cell viability tests were performed using the MTT colorimetric method. On the other hand, protein expression assays of two proteins involved in the signaling pathway of cell apoptosis Caspase- 3 cleaved (cas- 3 clv) and PARP-1 are carried out. There are statistically significant differences $(p<0,05)$ in the cell viability tests between Biodentine and control group, as well as the exposure time increases $(p<0,05)$. Otherwise, there are also significant differences $(p<0,05)$ in the expression of PARP-1 in the groups, sometimes a Biodentine. The results in this study that Biodentine generates a cellular cytotoxicity in HDPC cultures, therefore, cell viability as the expression of apoptotic proteins. This is why the use of this biomaterial should be studied for each particular clinical case, especially as a direct pulp capping agent.

KEY WORDS: cytotoxicity, biodentine, caspase 3 , poly(adp-ribose) polymerase, dental pulp cells.

\section{REFERENCIAS BIBLIOGRÁFICAS}

A Saberi, E.; Farhadmollashahi, N.; Ghotbi, F.; Karkeabadi, H. \& Havaei, R. Cytotoxic effects of mineral trioxide aggregate, calcium enrichedmixture cement, Biodentine and octacalcium pohosphate onhuman gingival fibroblasts. J. Dent. Res. Dent. Clin. Dent. Prospects, 10(2):75-80, 2016. 
Akbulut, M. B.; Arpaci, P. U. \& Eldeniz, A. U. Effects of four novel root-end filling materials on the viability of periodontal ligament fibroblasts. Restor. Dent. Endod., 43(3):e24, 2018.

Bortoluzzi, E. A.; Niu, L. N.; Palani, C. D.; El-Awady, A. R.; Hammond, B. D.; Pei, D. D.; Tian, F. C.; Cutler, C. W.; Pashley, D. H. \& Tay, F. R. Cytotoxicity and osteogenic potential of silicate calcium cements as potential protective materials for pulpal revascularization. Dent. Mater., 31(12):1510-22, 2015.

Boulares, A. H.; Yakovlev, A. G.; Ivanova, V.; Stoica, B. A.; Wang, G.; Iyer, S. \& Smulson, M. Role of poly(ADP-ribose) polymerase (PARP) cleavage in apoptosis. Caspase 3-resistant PARP mutant increases rates of apoptosis in transfected cells. J. Biol. Chem., 274(33):22932-40, 1999.

Camilleri, J.; Sorrentino, F. \& Damidot, D. Investigation of the hydration and bioactivity of radiopacified tricalcium silicate cement, Biodentine and MTA Angelus. Dent. Mater., 29(5):58093, 2013.

Chang, S. W.; Bae, W. J.; Yi, J. K.; Lee, S.; Lee, D. W.; Kum, K. Y. \& Kim, E. C. Odontoblastic differentiation, inflammatory response, and angiogenic potential of 4 calcium silicate-based cements: Micromega MTA, ProRoot MTA, RetroMTA, and Experimental Calcium Silicate Cement. J. Endod., 41(9):1524-9, 2015.

Chang, S. W.; Lee, S. Y.; Ann, H. J.; Kum, K. Y. \& Kim, E. C. Effects of calcium silicate endodontic cements on biocompatibility and mineralization-inducing potentials in human dental pulp cells. $J$. Endod., 40(8):1194-200, 2014.

Cheng, Y. Y.; Yang, J. S.; Tsai, S. C.; Liaw, C. C.; Chung, J. G.; Huang, L. J.; Lee, K. H.; Lu, C. C.; Chien, H. C.; Tsuzuki, M.; et al. The newly synthesized 2-(3-hydroxy-5-methoxyphenyl)-6,7methylenedioxyquinolin-4-one triggers cell apoptosis through induction of oxidative stress and upregulation of the p38 MAPK signaling pathway in HL-60 human leukemia cells. Oncol. Rep., 28(4):1482-90, 2012.

Emara, R.; Elhennawy, K. \& Schwendicke, F. Effects of calcium silicate cements on dental pulp cells: A systematic review. J. Dent., 77:18-36, 2018.

Fragoso, J. M.; Alarcón, G. V.; Morales, S. J.; Hernández, O. D. R. \& Bello, J. R. El factor de necrosis tumoral a (TNF-a) en las enfermedades autoinmunes (EA): biología molecular y genética. Gac. Med. Mex., 150:334-44, 2014.

Jung, J. Y.; Woo, S. M.; Lee, B. N.; Koh, J. T.; Nör, J. E. \& Hwang, Y. C. Effect of Biodentine and Bioaggregate on odontoblastic differentiation via mitogen-activated protein kinase pathway in human dental pulp cells. Int. Endod. J., 48(2):174-84, 2015.

Li, W.; Zhou, J. \& Xu, Y. Study of the in vitro cytotoxicity testing of medical devices. Biomed. Rep., 3(5):617-20, 2015.

Nikitakis, N. G.; Sauk, J. J. \& Papanicolaou, S. I. The role of apoptosis in oral disease: mechanisms; aberrations in neoplastic, autoimmune, infectious, hematologic, and developmental diseases; and therapeutic opportunities. Oral Surg. Oral Med. Oral Pathol. Oral Radiol. Endod., 97(4):476-90, 2004.

Ozdemir, H. O.; Ozçelik, B.; Karabucak, B. \& Cehreli, Z. C. Calcium ion diffusion from mineral trioxide aggregate through simulated root resorption defects. Dent. Traumatol., 24(1):70-3, 2008.

Rathinam, E.; Rajasekharan, S.; Chiturri, R. T.; Declercq, H.; Martens, L. \& De Coster, P. Gene expression profiling and molecular signaling of various cells in response to tricalcium silicate cements: A systematic review. J. Endod., 42(12):1713-25, 2016.

Simon, H. U.; Haj-Yehia, A. \& Levi-Schaffer, F. Role of reactive oxygen species (ROS) in apoptosis induction. Apoptosis, 5(5):415-8, 2000 ,

Sosna, J.; Voigt, S.; Mathieu, S.; Lange, A.; Thon, L.; Davarnia, P.; Herdegen, T.; Linkermann, A.; Rittger, A.; Chan, F.; et al. TNFinduced necroptosis and PARP-1-mediated necrosis represent distinct routes to programmed necrotic cell death. Cell. Mol. Life Sci., 71(2):331-48, 2014.
Wang, Z. Q.; Stingl, L.; Morrison, C.; Jantsch, M.; Los, M.; SchulzeOsthoff, K. \& Wagner, E. F. PARP is important for genomic stability but dispensable in apoptosis. Genes Dev., 11(18):2347-58, 2015.

Zhou, H. M.; Shen, Y.; Wang, Z. J.; Li, L.; Zheng, Y. F.; Häkkinen, L. \& Haapasalo, M. In vitro cytotoxicity evaluation of a novel root repair material. J. Endod., 39(4):478-83, 2013.

Dirección para correspondencia:

Pablo Romero

Los Raulíes \# 425 Isla Teja

Valdivia

CHILE

Email: pabloromeroaraya@gmail.com.

Recibido : 22-05-2019

Aceptado: 25-06-2019 